\title{
An Efficient Algorithm for Constructing all Magic Squares of Order Four
}

\author{
Changyu Liu ${ }^{1,4}$, Tiezhu Zhao ${ }^{2, *}$ and Bin $\mathrm{Lu}^{3}$ \\ ${ }^{1}$ College of Mathematics and Informatics, South China Agricultural University, \\ Guangzhou 510642, China \\ ${ }^{2}$ Computer College, Dongguan University of Technology, Dongguan 523808, China \\ ${ }^{3}$ School of Computer Science, Wuyi University, Jiangmen 529020, China \\ ${ }^{4}$ School of Computer Science and Engineering, South China University of \\ Technology, Guangzhou 510006, China \\ yezhich@gmail.com, tzzhao83@163.com,lbscut@gmail.com
}

\begin{abstract}
Like the Sudoku puzzle, the magic square involves recreational mathematical contexts and has attracted considerable attention. Although there are many researches available, few of them could provide an efficient solution for constructing all magic squares of order four. In this paper, we firstly formulate constraints of magic squares of order four by a collection of linear equations and provide an intuitive but computationally infeasible solution to these equations. Then, we propose an efficient algorithm for constructing all magic squares of order four. The algorithm transforms solving these equations into finding all possible permutations of seven free variables from sixteen consecutive natural integers based on the Gaussian Elimination method. Furthermore, we show the effectiveness of the proposed algorithm by 48 magic squares in the experimental section.
\end{abstract}

Keywords: efficient algorithm, magic square, order four, permutations, Gaussian Elimination

\section{Introduction}

As the ancestor of Sudoku, the magic square of order $n$ is an $n$-by- $n$ matrix of numbers, where the sum of numbers along each row, each column, the forward main diagonal and the backward main diagonal are the same constant $\mu$ which is also called the magic sum [1]. A magic square that contains integers from 1 to $n^{2}$ is called a classical magic square or natural magic square [3] with $\mu=n\left(n^{2}+1\right) / 2$.

The research on magic squares has a long rich history. Involving both mathematical contexts and philosophical or religious contexts, magic squares have been firstly found in Chinese literature written about A. D. 1125 [5]. Despite a long rich history, magic squares are still the subject of research projects. For example, in paper [7], magic squares are combined with the historical research, whereas in $[9,11]$, magic squares are connected with the pure mathematical research, such as the algebraic and combinatorial geometry of polyhedral.

The purpose of the study is to construct all magic squares of order four. Although there are many researches available, few of them could provide an efficient solution for constructing all magic squares of order four. In this paper, we firstly introduce a collection of linear equations to formulate the desired problem. Then, we provide an efficient optimization method for all magic squares of order four by adopting the well

* Corresponding Author 
known Gaussian Elimination algorithm to solve these equations. At last, we show the effectiveness of the algorithm by several experimental results.

\section{Magic Squares of Order Four}

The magic square of order four is a 4 by 4 matrix, which contains integers $\{1,2, \cdots, 16\}$. The task for constructing all magic squares of order four is to find all possible permutations of integers $\{1,2, \cdots, 16\}$, so that the magic sum of the square is $\mu=$ $4\left(4^{2}+1\right) / 2=34$. Figure 1 shows the fourth order magic square puzzle, where Figure 1(a) shows ten constraints, i.e. all s1 to s10 should be 34, and Figure 1(b) shows such an example.

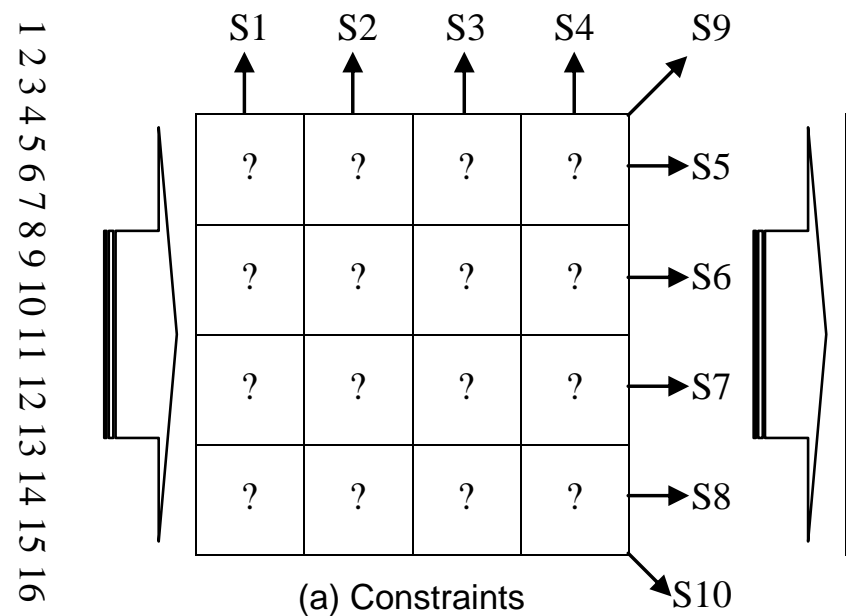

(a) Constraints

\begin{tabular}{|c|c|c|c|}
\hline 8 & 14 & 3 & 9 \\
\hline 2 & 15 & 10 & 7 \\
\hline 13 & 4 & 5 & 12 \\
\hline 11 & 1 & 16 & 6 \\
\hline
\end{tabular}

(b) An example

Figure 1. Fourth Order Magic Square Puzzle

Let $\left\{x_{1}, x_{2}, x_{3}, \cdots, x_{16}\right\}$ be a permutation from $\{1,2, \cdots, 16\}$. The constructing process of all magic squares of order four, as shown in Figure 1, can be formulated in Figure 2.

\begin{tabular}{|l|l|l|l|}
\hline$x_{1}$ & $x_{2}$ & $x_{3}$ & $x_{4}$ \\
\hline$x_{5}$ & $x_{6}$ & $x_{7}$ & $x_{8}$ \\
\hline$x_{9}$ & $x_{10}$ & $x_{11}$ & $x_{12}$ \\
\hline$x_{13}$ & $x_{14}$ & $x_{15}$ & $x_{16}$ \\
\hline
\end{tabular}

Figure 2. Problem Formulation

Then, ten constraints of Figure 1(a) can be rewritten by ten equations, as

$$
\begin{gathered}
x_{1}+x_{2}+x_{3}+x_{4}=34 \\
x_{5}+x_{6}+x_{7}+x_{8}=34 \\
x_{9}+x_{10}+x_{11}+x_{12}=34 \\
x_{13}+x_{14}+x_{15}+x_{16}=34
\end{gathered}
$$




$$
\begin{aligned}
& x_{1}+x_{5}+x_{9}+x_{13}=34 \\
& x_{2}+x_{6}+x_{10}+x_{14}=34 \\
& x_{3}+x_{7}+x_{11}+x_{15}=34 \\
& x_{4}+x_{8}+x_{12}+x_{16}=34 \\
& x_{1}+x_{6}+x_{11}+x_{16}=34 \\
& x_{4}+x_{7}+x_{10}+x_{13}=34
\end{aligned}
$$

Accordingly, the task to construct all magic squares of order four is to find all possible solutions for above ten equations.

\section{An Infeasible Solution}

One intuitive solution to above equations is to use the exhaustive search method, as shown in Algorithm 1.

Algorithm 1. An exhaustive search method for all magic squares of order four

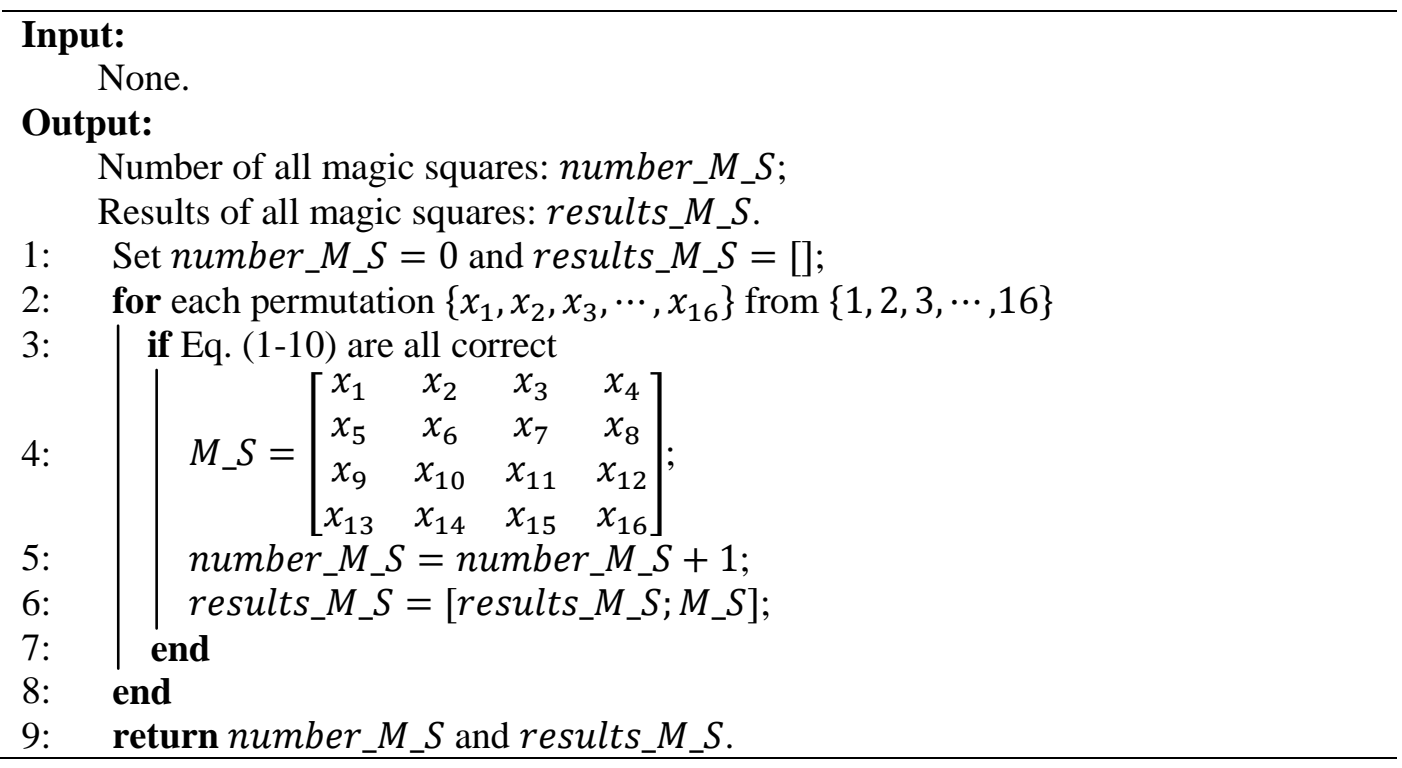

However, this is an infeasible solution. Because, for the major loop, the algorithm would compute 16 ! times, which is computationally expensive.

\section{Methodology}

In this section, we provide an efficient solution.

\subsection{Gaussian Elimination}

Firstly, we use the Gaussian Elimination method to reformulate Eq. (1-10). According to Eq. (5-8), we obtain

$$
\begin{aligned}
& x_{13}=34-x_{1}-x_{5}-x_{9} \\
& x_{14}=34-x_{2}-x_{6}-x_{10}
\end{aligned}
$$




$$
\begin{aligned}
& x_{15}=34-x_{3}-x_{7}-x_{11} \\
& x_{16}=34-x_{4}-x_{8}-x_{12}
\end{aligned}
$$

According to Eq. (11-14), we have

$$
x_{13}+x_{14}+x_{15}+x_{16}=4 \times 34-\left(x_{1}+x_{2}+\cdots+x_{12}\right)
$$

Substitute Eq. (1-3) into Eq. (15), we get

$$
x_{13}+x_{14}+x_{15}+x_{16}=34
$$

This means that Eq. (4) is redundant. Then, substitute Eq. (14) into Eq. (9) and Eq. (11) into Eq. (10), we acquire

$$
\begin{aligned}
& x_{12}=x_{1}+x_{6}+x_{11}-x_{4}-x_{8} \\
& x_{10}=x_{1}+x_{5}+x_{9}-x_{4}-x_{7}
\end{aligned}
$$

Substitute Eq. (16-17) into Eq. (3), we have

$$
x_{11}=\frac{1}{2}\left(2 x_{4}+x_{7}+x_{8}-2 x_{1}-x_{5}-x_{6}-2 x_{9}+34\right)
$$

Furthermore, we can derive from Eq. (1) that

$$
\begin{aligned}
& x_{4}=34-x_{1}-x_{2}-x_{3} \\
& x_{8}=34-x_{5}-x_{6}-x_{7}
\end{aligned}
$$

Thus, variables $\left\{x_{4}, x_{8}, x_{10}, x_{11}, x_{12}, x_{13}, x_{14}, x_{15} x_{16}\right\}$ can be determined by free variables $\left\{x_{1}, x_{2}, x_{3}, x_{5}, x_{6}, x_{7}, x_{9}\right\}$. Thereby, the task to construct all magic squares of order four is transformed into finding all possible permutations of $\left\{x_{1}, x_{2}, x_{3}, x_{5}, x_{6}, x_{7}, x_{9}\right\}$ from $\{1,2,3, \cdots, 16\}$.

\subsection{Optimization Algorithm}

Secondly, we provide an efficient optimization method for all magic squares of order four, as shown in Algorithm 2. 
Algorithm 2. An efficient optimization method for all magic squares of order four

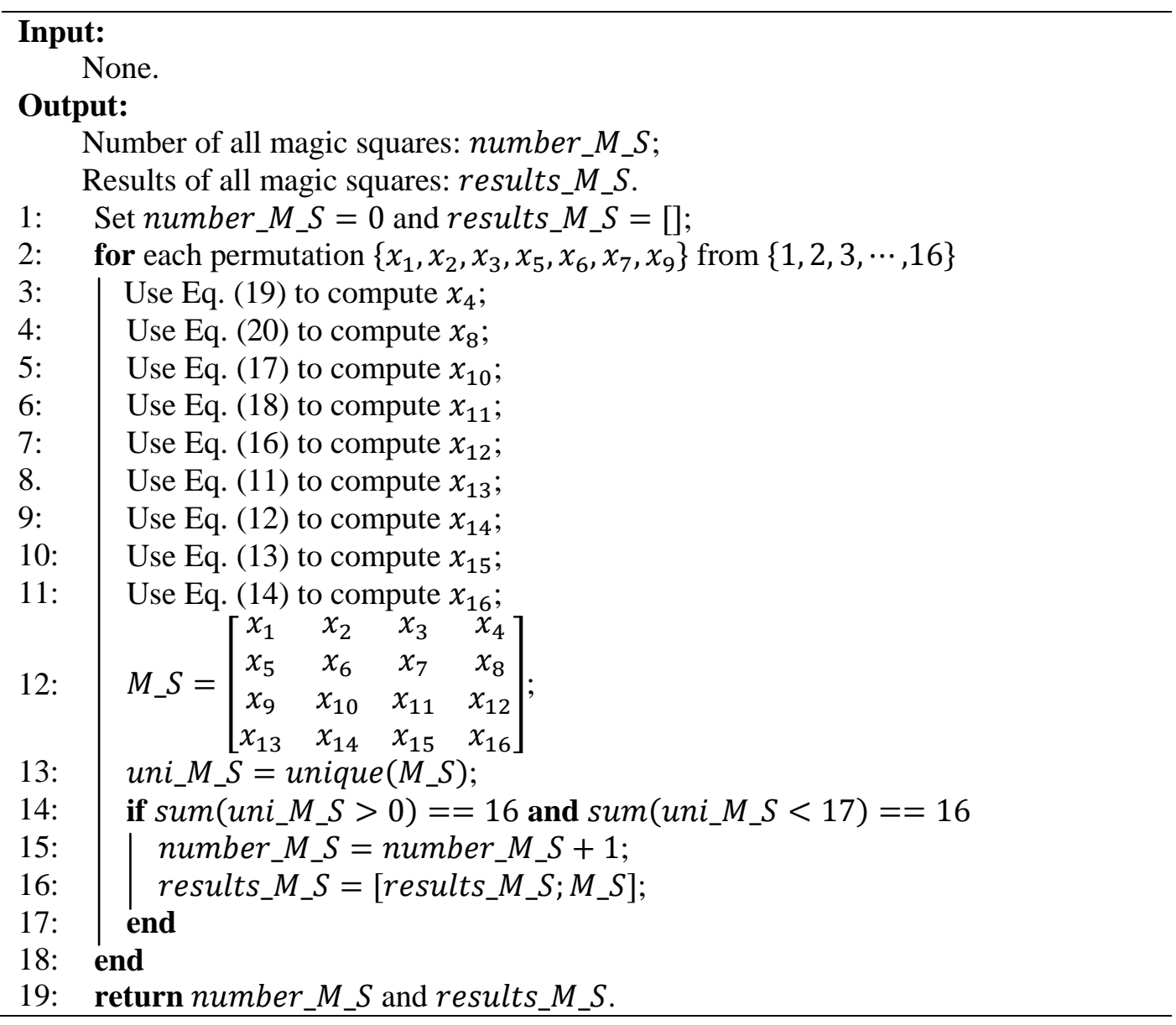

The loop from line 2 to line 18 implements $P_{16}^{7}$ iterations for constructing all magic squares of order four by using the Gaussian Elimination method, where unique $\left(M_{-} S\right.$ ) returns a vector which contains the same value as in $M_{-} S$ but no repetitions, and $\operatorname{sum}(\cdot)$ returns sums along different dimensions of an array. Compared to the Algorithm 1, the proposed Algorithm 2 is $362880\left(16 ! / P_{16}^{7}=9\right.$ !) times faster, where we take the complexities inside major loops of the two algorithms to be simply all $O(1)$. So, the Algorithm 2 is very efficient.

\section{Experiment}

We develop the Algorithm 2 in Matlab R2010a. Besides, all experiments are implemented under the Windows 7 Operating System, 4 GB RAM and Intel ${ }^{\circledR}$ Core ${ }^{\mathrm{TM}} 2$ Duo CPU. It takes about 45 minutes to get all the 7040 unique magic squares of order four, which is coincident with results in $[13,15,17]$. Due to the limited space, we show only the first 24 and the last 24 magic squares of order four in Figure 3 and Figure 4 separately. 


\begin{tabular}{|c|c|c|c|}
\hline 1 & 2 & 15 & 16 \\
\hline 12 & 14 & 3 & 5 \\
\hline 13 & 7 & 10 & 4 \\
\hline 8 & 11 & 6 & 9 \\
\hline \multicolumn{4}{|c|}{0001}
\end{tabular}

\begin{tabular}{|c|c|c|c|}
\hline 1 & 3 & 14 & 16 \\
\hline 12 & 13 & 4 & 5 \\
\hline 15 & 8 & 9 & 2 \\
\hline 6 & 10 & 7 & 11 \\
\hline \multicolumn{4}{|c|}{0005} \\
\hline
\end{tabular}

\begin{tabular}{|c|c|c|c|}
\hline 1 & 3 & 16 & 14 \\
\hline 12 & 15 & 2 & 5 \\
\hline 13 & 10 & 7 & 4 \\
\hline 8 & 6 & 9 & 11 \\
\hline
\end{tabular}
0009

\begin{tabular}{|c|c|c|c|}
\hline 1 & 4 & 13 & 16 \\
\hline 8 & 15 & 2 & 9 \\
\hline 14 & 5 & 12 & 3 \\
\hline 11 & 10 & 7 & 6 \\
\hline \multicolumn{4}{|c|}{0013} \\
\end{tabular}

\begin{tabular}{|c|c|c|c|}
\hline 1 & 4 & 13 & 16 \\
\hline 14 & 15 & 2 & 3 \\
\hline 12 & 9 & 8 & 5 \\
\hline 7 & 6 & 11 & 10 \\
\hline
\end{tabular}
0017

\begin{tabular}{|c|c|c|c|}
\hline 1 & 4 & 14 & 15 \\
\hline 13 & 16 & 2 & 3 \\
\hline 8 & 5 & 11 & 10 \\
\hline 12 & 9 & 7 & 6 \\
\hline \multicolumn{4}{|c|}{0021}
\end{tabular}

\begin{tabular}{|c|c|c|c|}
\hline 1 & 2 & 15 & 16 \\
\hline 13 & 14 & 3 & 4 \\
\hline 12 & 7 & 10 & 5 \\
\hline 8 & 11 & 6 & 9 \\
\hline \multicolumn{4}{|c|}{0002}
\end{tabular}

\begin{tabular}{|c|c|c|c|}
\hline 1 & 2 & 16 & 15 \\
\hline 13 & 14 & 4 & 3 \\
\hline 12 & 7 & 9 & 6 \\
\hline 8 & 11 & 5 & 10 \\
\hline \multicolumn{4}{|c|}{0003}
\end{tabular}

\begin{tabular}{|c|c|c|c|}
\hline 1 & 3 & 14 & 16 \\
\hline 10 & 13 & 4 & 7 \\
\hline 15 & 6 & 11 & 2 \\
\hline 8 & 12 & 5 & 9 \\
\hline \multicolumn{4}{|c|}{0004}
\end{tabular}

\begin{tabular}{|c|c|c|c|}
\hline 1 & 3 & 14 & 16 \\
\hline 15 & 13 & 4 & 2 \\
\hline 10 & 6 & 11 & 7 \\
\hline 8 & 12 & 5 & 9 \\
\hline \multicolumn{4}{|c|}{0006}
\end{tabular}

\begin{tabular}{|c|c|c|c|}
\hline 1 & 3 & 14 & 16 \\
\hline 15 & 13 & 4 & 2 \\
\hline 12 & 8 & 9 & 5 \\
\hline 6 & 10 & 7 & 11 \\
\hline \multicolumn{4}{|c|}{0007}
\end{tabular}

\begin{tabular}{|c|c|c|c|}
\hline 1 & 3 & 16 & 14 \\
\hline 13 & 15 & 2 & 4 \\
\hline 8 & 6 & 11 & 9 \\
\hline 12 & 10 & 5 & 7 \\
\hline \multicolumn{4}{|c|}{0010}
\end{tabular}

\begin{tabular}{|c|c|c|c|}
\hline 1 & 3 & 16 & 14 \\
\hline 13 & 15 & 2 & 4 \\
\hline 12 & 10 & 7 & 5 \\
\hline 8 & 6 & 9 & 11 \\
\hline \multicolumn{4}{|c|}{0011}
\end{tabular}

\begin{tabular}{|c|c|c|c|}
\hline 1 & 3 & 16 & 14 \\
\hline 8 & 15 & 2 & 9 \\
\hline 13 & 6 & 11 & 4 \\
\hline 12 & 10 & 5 & 7 \\
\hline \multicolumn{4}{|c|}{0008} \\
\end{tabular}

\begin{tabular}{|c|c|c|c|}
\hline 1 & 4 & 13 & 16 \\
\hline 8 & 14 & 3 & 9 \\
\hline 15 & 5 & 12 & 2 \\
\hline 10 & 11 & 6 & 7 \\
\hline \multicolumn{4}{|c|}{0012}
\end{tabular}

\begin{tabular}{|c|c|c|c|}
\hline 1 & 4 & 13 & 16 \\
\hline 12 & 14 & 3 & 5 \\
\hline 15 & 9 & 8 & 2 \\
\hline 6 & 7 & 10 & 11 \\
\hline
\end{tabular}

\begin{tabular}{|c|c|c|c|}
\hline 1 & 4 & 13 & 16 \\
\hline 15 & 14 & 3 & 2 \\
\hline 8 & 5 & 12 & 9 \\
\hline 10 & 11 & 6 & 7 \\
\hline \multicolumn{4}{|c|}{0018}
\end{tabular}

\begin{tabular}{|c|c|c|c|}
\hline 1 & 4 & 13 & 16 \\
\hline 12 & 15 & 2 & 5 \\
\hline 14 & 9 & 8 & 3 \\
\hline 7 & 6 & 11 & 10 \\
\hline
\end{tabular}
0015

\begin{tabular}{|c|c|c|c|}
\hline 1 & 4 & 13 & 16 \\
\hline 15 & 14 & 3 & 2 \\
\hline 12 & 9 & 8 & 5 \\
\hline 6 & 7 & 10 & 11 \\
\hline \multicolumn{4}{|c|}{0019}
\end{tabular}

\begin{tabular}{|c|c|c|c|}
\hline 1 & 4 & 14 & 15 \\
\hline 13 & 16 & 2 & 3 \\
\hline 12 & 9 & 7 & 6 \\
\hline 8 & 5 & 11 & 10 \\
\hline \multicolumn{4}{|c|}{0022}
\end{tabular}

\begin{tabular}{|c|c|c|c|}
\hline 1 & 4 & 14 & 15 \\
\hline 16 & 11 & 5 & 2 \\
\hline 9 & 6 & 12 & 7 \\
\hline 8 & 13 & 3 & 10 \\
\hline \multicolumn{4}{|c|}{0023}
\end{tabular}

\begin{tabular}{|c|c|c|c|}
\hline 1 & 4 & 13 & 16 \\
\hline 14 & 15 & 2 & 3 \\
\hline 8 & 5 & 12 & 9 \\
\hline 11 & 10 & 7 & 6 \\
\hline \multicolumn{4}{|c|}{0016}
\end{tabular}

\begin{tabular}{|c|c|c|c|}
\hline 1 & 4 & 14 & 15 \\
\hline 9 & 12 & 6 & 7 \\
\hline 16 & 5 & 11 & 2 \\
\hline 8 & 13 & 3 & 10 \\
\hline \multicolumn{4}{|c|}{0020}
\end{tabular}

\begin{tabular}{|c|c|c|c|}
\hline 1 & 4 & 14 & 15 \\
\hline 16 & 13 & 3 & 2 \\
\hline 7 & 6 & 12 & 9 \\
\hline 10 & 11 & 5 & 8 \\
\hline \multicolumn{4}{|c|}{0024}
\end{tabular}

Figure 3. First 24 Magic Squares of Order Four 


\begin{tabular}{|c|c|c|c|c|c|c|c|c|c|c|c|c|c|c|c|}
\hline 16 & 13 & 3 & 2 & 16 & 13 & 3 & 2 & 16 & 13 & 3 & 2 & 16 & 13 & 3 & 2 \\
\hline 1 & 4 & 14 & 15 & 1 & 6 & 12 & 15 & 4 & 1 & 15 & 14 & 4 & 1 & 15 & 14 \\
\hline 10 & 11 & 5 & 8 & 8 & 11 & 5 & 10 & 5 & 8 & 10 & 11 & 9 & 12 & 6 & 7 \\
\hline 7 & 6 & 12 & 9 & 9 & 4 & 14 & 7 & 9 & 12 & 6 & 7 & 5 & 8 & 10 & 11 \\
\hline \multicolumn{4}{|c|}{7017} & \multicolumn{4}{|c|}{7018} & \multicolumn{4}{|c|}{7019} & \multicolumn{4}{|c|}{7020} \\
\hline 16 & 13 & 3 & 2 & 16 & 13 & 4 & 1 & 16 & 13 & 4 & 1 & 16 & 13 & 4 & 1 \\
\hline 8 & 5 & 11 & 10 & 2 & 3 & 14 & 15 & 2 & 3 & 14 & 15 & 3 & 2 & 15 & 14 \\
\hline 1 & 12 & 6 & 15 & 5 & 8 & 9 & 12 & 9 & 12 & 5 & 8 & 5 & 8 & 9 & 12 \\
\hline 9 & 4 & 14 & 7 & 11 & 10 & 7 & 6 & 7 & 6 & 11 & 10 & 10 & 11 & 6 & 7 \\
\hline \multicolumn{4}{|c|}{7021} & \multicolumn{4}{|c|}{7022} & \multicolumn{4}{|c|}{7023} & \multicolumn{4}{|c|}{7024} \\
\hline 16 & 13 & 4 & 1 & 16 & 13 & 4 & 1 & 16 & 13 & 4 & 1 & 16 & 13 & 4 & 1 \\
\hline 3 & 2 & 15 & 14 & 5 & 2 & 15 & 12 & 5 & 3 & 14 & 12 & 9 & 2 & 15 & 8 \\
\hline 9 & 12 & 5 & 8 & 3 & 8 & 9 & 14 & 2 & 8 & 9 & 15 & 3 & 12 & 5 & 14 \\
\hline 6 & 7 & 10 & 11 & 10 & 11 & 6 & 7 & 11 & 10 & 7 & 6 & 6 & 7 & 10 & 11 \\
\hline \multicolumn{4}{|c|}{7025} & \multicolumn{4}{|c|}{7026} & \multicolumn{4}{|c|}{7027} & \multicolumn{4}{|c|}{7028} \\
\hline 16 & 13 & 4 & 1 & 16 & 14 & 1 & 3 & 16 & 14 & 1 & 3 & 16 & 14 & 1 & 3 \\
\hline 9 & 3 & 14 & 8 & 4 & 2 & 15 & 13 & 4 & 2 & 15 & 13 & 5 & 2 & 15 & 12 \\
\hline 2 & 12 & 5 & 15 & 5 & 7 & 10 & 12 & 9 & 11 & 6 & 8 & 4 & 7 & 10 & 13 \\
\hline 7 & 6 & 11 & 10 & 9 & 11 & 8 & 6 & 5 & 7 & 12 & 10 & 9 & 11 & 8 & 6 \\
\hline \multicolumn{4}{|c|}{7029} & \multicolumn{4}{|c|}{7030} & \multicolumn{4}{|c|}{7031} & \multicolumn{4}{|c|}{7032} \\
\hline 16 & 14 & 1 & 3 & 16 & 14 & 3 & 1 & 16 & 14 & 3 & 1 & 16 & 14 & 3 & 1 \\
\hline 9 & 2 & 15 & 8 & 2 & 4 & 13 & 15 & 2 & 4 & 13 & 15 & 5 & 4 & 13 & 12 \\
\hline 4 & 11 & 6 & 13 & 5 & 9 & 8 & 12 & 7 & 11 & 6 & 10 & 2 & 9 & 8 & 15 \\
\hline 5 & 7 & 12 & 10 & 11 & 7 & 10 & 6 & 9 & 5 & 12 & 8 & 11 & 7 & 10 & 6 \\
\hline \multicolumn{4}{|c|}{7033} & \multicolumn{4}{|c|}{7034} & \multicolumn{4}{|c|}{7035} & \multicolumn{4}{|c|}{7036} \\
\hline 16 & 14 & 3 & 1 & 16 & 15 & 1 & 2 & 16 & 15 & 2 & 1 & 16 & 15 & 2 & 1 \\
\hline 7 & 4 & 13 & 10 & 4 & 3 & 13 & 14 & 4 & 3 & 14 & 13 & 5 & 3 & 14 & 12 \\
\hline 2 & 11 & 6 & 15 & 5 & 10 & 8 & 11 & 5 & 10 & 7 & 12 & 4 & 10 & 7 & 13 \\
\hline 9 & 5 & 12 & 8 & 9 & 6 & 12 & 7 & 9 & 6 & 11 & 8 & 9 & 6 & 11 & 8 \\
\hline
\end{tabular}

Figure 4. Last 24 Magic Squares of Order Four 


\section{Conclusion}

In this paper, we proposed an efficient algorithm, which is much faster than the infeasible exhaustive search method, for constructing all magic squares of order four by using the Gaussian Elimination method to solve a collection of formulated linear equations. In the experiment section, we show the first 24 magic squares and the last 24 magic squares of the algorithm. In the future, we would like to develop a similar efficient algorthm for constructing all magic squares of order five.

\section{Acknowledgments}

This paper was supported by the National Natural Science Foundation of China under Grant No. 61402106, the Natural Science Foundation of Guangdong Province, China under Grant No. 2014A030313632, and the Doctor Startup Foundation of Wuyi University under Grant No. 2014BS07. We would like to thank anonymous reviewers for helpful comments.

\section{References}

[1]. J. P. Delahaye, "The science behind Sudoku," Sci. Am., vol. 294, no. 6, (2006), pp. 80-87.

[2]. W. Li, et al., "A construction for doubly pandiagonal magic squares," Discrete Math., vol. 312, no. 2, (2012), pp. 479-485.

[3]. M. Z. Lee, et al., "On nonsingular regular magic squares of odd order," Linear Algebra Appl., vol. 437, no. 6, (2012), pp. 1346-1355.

[4]. H. Wang, et al., "Study on behavior simulation for picking manipulator in virtual environment based on binocular stereo vision," In ICSC, (2008), pp. 27-31.

[5]. W. S. Andrews, "Magic squares and cubes," Cosimo, Inc., (2004).

[6]. J. X. Chen, et al., "Modeling and performance analyzing of helix transmission base on Modelica," Key Eng. Mater., vol. 455, (2011), pp. 511-515.

[7]. P. C. Pasles, "The lost squares of Dr. Franklin: Ben Franklin's missing squares and the secret of the magic circle," Am. Math. Mon., (2001), pp. 489-511.

[8]. H. Li, et al., "One-Step heterogeneous catalytic process for the dehydration of xylan into furfural," BioResour., vol. 8, no. 3, (2013), pp. 3200-3211.

[9]. M. Ahmed, et al., "Polyhedral cones of magic cubes and squares," In Discrete Comput. Geom., Springer Berlin Heidelberg, (2003), pp. 25-41.

[10]. C. Liu, et al., "Secure access control and large scale robust representation for online multimedia event detection," Sci. World J., vol. 2014, Article ID 219732, (2014), 12 pages.

[11]. M. Beck and D. Pixton, "The Ehrhart polynomial of the Birkhoff polytope," Discrete Comput. Geom., vol. 30, no. 4, (2003), pp. 623-637.

[12]. R. P. Nordgren, "New constructions for special magic squares," Int. J. Pure Appl. Math., vol. 2, no. 2, (2012), pp. 133-154.

[13]. S. Al-Ashhab, "Special magic squares of order xix and eight," Int. J. Digit. Inf. Wirel. Commun., vol. 1, no. 4, (2011), pp. 733-745.

[14]. B. Lu, et al., "Discovery of community structure in complex networks based on resistance distance and center nodes," J. Comput. Inf. Syst., vol. 8, no. 23, (2012), pp. 9807-9814.

[15]. J. B. Da Fonseca, "The magic square as a benchmark: comparing manual solution with mip solution and AI algorithm and improved evolutionary algorithm," WSEAS T. Inf. Sci. Appl., vol. 2, no. 5, (2005), pp. 486-494.

[16]. D. Xu, et al., "New recursive construction of magic squares using Kronecker compositional operations and its application in engineering computation," Sys. Eng. Procedia, vol. 2, (2011), pp. 331-337.

[17]. K. O. D. D. DL and C. H. FIMA, "Constructing pandiagonal magic squares of arbitrarily large size," Math. Today, (2006), pp. 23-29.

[18]. H. L. Li, et al., "Catalytic hydrothermal pretreatment of corncob into xylose and furfural via solid acid catalyst," Biore. Tech., vol. 158, (2014), pp. 313-320.

[19]. R. P. Nordgren, "On properties of special magic square matrices," Linear Algebr. Appl., vol. 437, no. 8, (2012), pp. 2009-2025.

[20]. C. Y. Liu, et al., "A hybrid simulation system for juicing and filling production line,” Int. J. Smart Home, vol. 8, no. 6, (2014), pp. 157-168.

[21]. D. B. Ojha and B. L. Kaul, "Generalization of $6 \times 6$ magic square," J. Educ. Vocat. Res., vol. 2, no. 1, (2011), pp. 18-23.

[22]. H. J. Wang, et al., "Study on a location method for bio-objects in virtual environment based on neural network and fuzzy reasoning," In ICIRA, (2009), pp. 1004-1012. 
[23]. K. S. Sreeranjini and V. M. Mallayya, "Semi magic squares as a field,” Int. J. Algebr., vol. 6, no. 26, (2012), pp. 1249-1256.

[24]. Y. H. Zhang, et al., "Robust segmentation of highly dynamic scene with missing data," IEICE T. Inf. Syst., vol. E98.D, no. 1, (2015), pp. 201-205.

[25]. K. S. Sreeranjini and V. M. Mallayya, "Morphisms between the groups of semi magic squares and real numbers," Int. J. Algebr., vol. 8, no. 19, (2014), pp. 903-907.

[26]. H. Li, et al., "Photocatalytic reduction of carbon dioxide to methanol by $\mathrm{Cu} 2 \mathrm{O} / \mathrm{SiC}$ nanocrystallite under visible light irradiation," J. Nat. Gas Chem., vol. 20, no. 2, (2011), pp. 145-150.

[27]. K. Chen, et al., "Regular sparse anti-magic squares with the second maximum density," Linear Algebr. Appl., vol. 457, (2014), pp. 12-28.

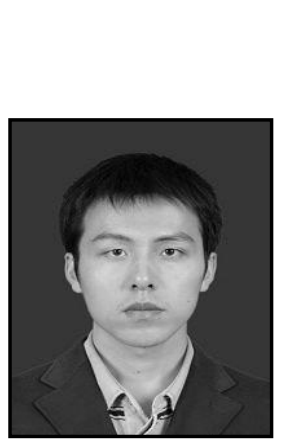

\section{Authors}

Changyu Liu received the $\mathrm{PhD}$ degree from South China University of Technology in 2015. He is currently a lecturer at South China Agricultural University. He was a visiting scholar at the School of Computer Science, Carnegie Mellon University, from September 2012 to October 2013, advised by Dr. Alex Hauptmann. Then, he worked with Prof. Mohamed Abdel-Mottaleb and Prof. Mei-Ling Shyu at the Department of Electrical and Computer Engineering, University of Miami, from October 2013 to September 2014. His research interests include computer vision and machine learning.

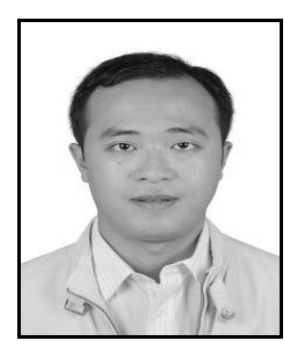

Tiezhu Zhao received the Ph.D. degree from South China University of Technology in 2011. He is currently a research associate at the Computer College, Dongguan University of Technology. His research interests include distributed/parallel computing, pattern recognition and network security.

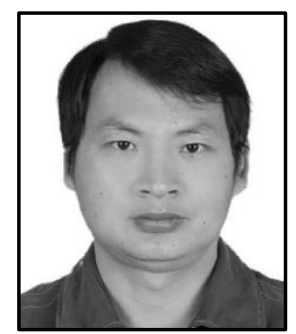

Bin Lu is currently a lecturer in the School of Computer Science at Wuyi University. He received his Ph.D. degree in 2013 from South China University of Technology. He is a reviewer of the Journal of Yangtze River Scientific Research Institute since 2010. His main research interests include complex network and machine learning. 
International Journal of Control and Automation Vol.8, No.11 (2015) 